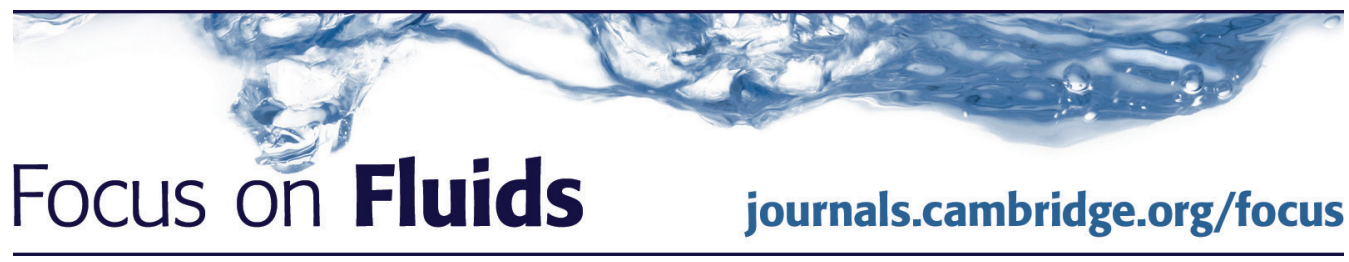

\title{
Inside the head and tail of a turbulent gravity current
}

\author{
Graham O. Hughes $\dagger$ \\ Research School of Earth Sciences, \\ The Australian National University, \\ Canberra ACT 2601, Australia
}

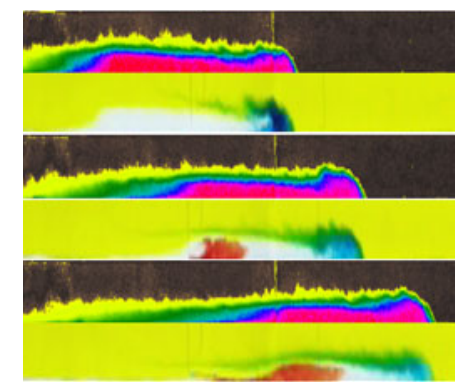

Gravity currents are an important buoyancy-driven flow in environmental, geophysical and industrial settings. Turbulence and mixing is commonplace in these flows, but is typically overlooked in theoretical models and predictions. Sher \& Woods (J. Fluid Mech., vol. 784, 2015, pp. 130-162) have quantified the velocity and density structure in turbulent gravity currents by combining high-quality experimental data with new theory. Their insights are set to stimulate significant advances in the area.

Key words: gravity currents, stratified flows, stratified turbulence

\section{Introduction}

Sea breezes, katabatic winds, thunderstorm outflows, avalanches, industrial gas spillages, estuarine salt intrusions, dam bursts, dust storms, pyroclastic flows and dense-slope plumes in the oceans are all examples of gravity currents. These flows form when buoyancy forces cause fluid of one density to encroach along a boundary into an ambient environment of different density (figure 1). The boundary can be inclined, but is often assumed to be horizontal. The typical structure of a gravity current consists of a bulbous 'head' and propagating front, where a strong density gradient between the two fluids is maintained, and a 'tail' extending back towards the initial source (figure $1 b$ ). Intense turbulence is often apparent in the vicinity of the head, and mass and tracers are transported laterally over a significant distance.

Early studies established the broad characteristics of gravity currents. Benjamin (1968) considered the momentum balance for an immiscible inviscid and steady current in a channel, predicting the front propagation speed and that energy must be dissipated in most cases. Studies since then focusing on the front speed have highlighted different dynamical regimes (summarized below for currents in which viscous drag at the boundary is unimportant). Following initial release of the current,

$\dagger$ Present address: Department of Civil and Environmental Engineering,

Imperial College London, London SW7 2AZ, UK.

Email address for correspondence: g.hughes@imperial.ac.uk 
(a)

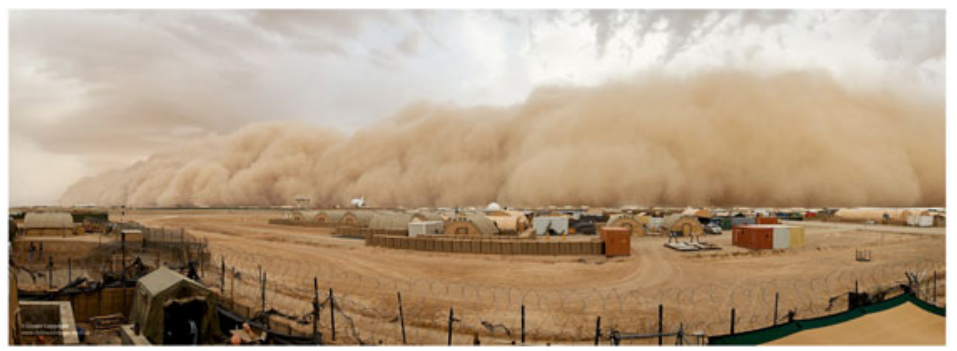

(b)

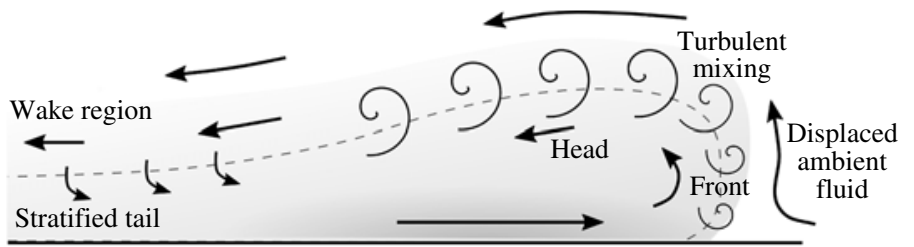

FIgURE 1. (a) Dust storm in Afghanistan (photograph: Cpl Daniel Wiepen; 45158327.jpg from defenceimages.mod.uk, Open Government License nationalarchives.gov.uk/doc/open-government-licence/; Crown Copyright 2014), (b) schematic diagram of flow structure in a vertical plane through a turbulent gravity current (delineated nominally by the dashed line; after figures $1(a)$ and 8 of Sher \& Woods (2015)). Density and velocity structure is indicated by shading and bold arrows, respectively.

the front speed is constant but at least $15 \%$ less than Benjamin's prediction (Huppert \& Simpson 1980; Shin, Dalziel \& Linden 2004), the suggested reason being the mismatch between the resulting velocity structure and that assumed in the theory. If a limited volume of source fluid is available to the current, the constant-speed regime (also known as the 'slumping' phase) persists until exhaustion of the source is communicated to the head (see Rottman \& Simpson 1983 for details). A second regime follows in which the current is well described by an approximately self-similar dynamical balance between buoyancy and inertia forces, and the front speed $u_{n}$ decays with time $t\left(u_{n} \sim t^{-1 / 3}\right.$, Huppert \& Simpson (1980)).

Turbulence in the vicinity of the gravity current head arises from instabilities owing to shear between the current and ambient flow and of the vorticity field generated by passage of the front over a no-slip boundary (leading to 'lobe and cleft' structures; Härtel, Meiburg \& Necker (2000)). Turbulent eddies exchange mass and momentum between the current and ambient fluid, and lead to mixing of the density field if the fluids are miscible. The turbulence results in additional drag on the current and dissipation of energy, but the dynamical importance of these effects is unclear (e.g. Shin et al. (2004) found that the propagation speed and the effective current depth are well predicted by an energy-conserving theory). Although many previous measurements have highlighted rich density and velocity structure in a turbulent gravity current (e.g. Hacker, Linden \& Dalziel 1996; Hallworth et al. 1996; Kneller, Bennett \& McCaffrey 1999; Shin et al. 2004; Marino, Thomas \& Linden 2005), most previous theoretical models have assumed a highly idealized form based on lateral and/or depth averaging of the structure. While these idealizations can capture some dynamics associated with mixing (because the total buoyancy anomaly in the flow is conserved; Huppert 2006), the internal structure of the current and its role in the overall flow remains an outstanding question. 


\section{Overview}

Sher \& Woods (2015) have advanced our understanding of gravity current dynamics with detailed measurements and theoretical modelling of the flow structure. They have considered the sudden release of dense fluid to form a turbulent current that propagates into a miscible ambient fluid over a large horizontal distance (up to 25 times the length of the initial source volume). Laboratory data suggest a fresh interpretation of the circulation in the current, the key observations being (see figure $1 b)$ :

(i) fluid that has the largest density contrast with the ambient is always found towards the base of the current head;

(ii) the fluid identified in (i) circulates towards the front from behind, i.e. its flow speed exceeds the front speed $u_{n}$;

(iii) strong turbulent mixing arises where the ambient fluid is displaced past the head and maintains shear instabilities on the current;

(iv) some mixed fluid in the 'wake' region is displaced backwards (both relative to the front and, to a lesser extent, in a stationary frame of reference);

(v) the density contrast in the head decays gradually as mixed fluid in (iv) is deposited into the stratified tail region and begins to be recirculated back to the head (as in (ii));

(vi) the current head height $h$ (about $38 \%$ of the total flow depth, based on a threshold concentration) and the proportion of displaced ambient fluid that is mixed into the current $(60-80 \%)$ are both insensitive to the flow parameters.

The emerging picture is of a current evolving towards a self-similar flow structure that is attenuated gradually by turbulent mixing. Unmixed source fluid is present in the head during the slumping phase, despite intense mixing there (see discussion by Hacker et al. (1996), Hallworth et al. (1996), Fragoso, Patterson \& Wettlaufer (2013)). Sher \& Woods (2015) have highlighted how the mixing and the recirculation (in (v)) accounts for creation of a strongly stratified tail region and a density contrast in the current that decreases with distance behind the head (see also Marino et al. (2005)). The ongoing recirculation of the least-dilute fluid in the current ((i) and (ii)) maintains a relatively sharp density gradient at the front. One interesting feedback is that this density gradient will act to stabilize the current head against shear instability, thus reducing both the level of turbulence that would otherwise be present and the attenuation rate of the overall flow structure. Sher \& Woods (2015) have found that the front is characterized by an approximately constant Froude number $F r=u_{n} /\left(g^{\prime} h\right)^{1 / 2} \approx 0.9$ (where $g^{\prime}$ is a depth-averaged reduced gravity), consistent with recent previous work (Shin et al. 2004; Marino et al. 2005). Interestingly, they have observed circulation speeds in (ii) that are approximately $35 \%$ faster than the front speed in the slumping phase, and much closer to those expected if energy was conserved in the flow (Benjamin 1968; see discussion in Härtel et al. 2000; Shin et al. 2004). This differential is indicative of the Reynolds stresses (owing to the turbulent eddies and entrainment) and inertial drag exerted at the head of the current, where the predominant dissipation of energy occurs.

Sher \& Woods (2015) have developed a new theoretical description of the velocity and density field in a gravity current by assuming that the dynamical influence of turbulent entrainment and mixing is concentrated at the head. Their predictions are a good match to the experimental data, and show that the structure and flow in the self-similar regime are well described by an approximate balance between buoyancy 
and inertia forces. Their work both helps provide a unified interpretation of previous literature and highlights the dynamics necessary to improve gravity current models for many applications.

\section{Future}

The observation of many gravity currents as highly turbulent suggests that they are complicated flows. Many existing theoretical models yield bulk predictions for currents with neglect of the effects associated with turbulence, including dissipation, mixing and the creation of velocity and density structure. Therefore, the new insights of Sher \& Woods (2015) will have significant benefits for applications that rely on accurate prediction of the passage of tracers, such as hazard management.

An important outstanding question concerns the behaviour of a gravity current in a deep ambient fluid. Benjamin (1968) showed that energy must be dissipated in this situation and, although an aim of several studies has been measuring the energetics associated with mixing (e.g. Shin et al. 2004; Fragoso et al. 2013), the energy budget for a current in a deep ambient fluid remains to be comprehensively understood. Furthermore, previous studies (e.g. Huppert \& Simpson 1980; Shin et al. 2004; Marino et al. 2005) have typically characterized the current in terms of the Froude number $F r=u_{n} /\left(g^{\prime} h\right)^{1 / 2}$ at the front, but this condition does not close the problem because both the front speed $u_{n}$ and the current height $h$ are unknown (Huppert 2006). Understanding the velocity and density structure in the current and ambient fluid (see also Marino et al. 2005) could offer an interesting avenue for progress on this problem.

\section{References}

Benjamin, T. B. 1968 Gravity currents and related phenomena. J. Fluid Mech. 31, 209-248.

Fragoso, A. T., Patterson, M. D. \& Wettlaufer, J. S. 2013 Mixing in gravity currents. J. Fluid Mech. 734, R2.

Hacker, J., Linden, P. F. \& DAlziel, S. B. 1996 Mixing in lock-release gravity currents. Dyn. Atmos. Oceans 24, 183-195.

Hallworth, M. A., Phillips, J., Huppert, H. E. \& Sparks, R. S. J. 1996 Entrainment into two-dimensional and axisymmetric turbulent gravity currents. J. Fluid Mech. 308, 289-312.

Härtel, C., Meiburg, E. \& Necker, F. 2000 Analysis and direct numerical simulation of the flow at a gravity-current head. Part 1. Flow topology and front speed for slip and no-slip boundaries. J. Fluid Mech. 418, 189-212.

Huppert, H. E. 2006 Gravity currents: a personal perspective. J. Fluid Mech. 554, 299-322.

Huppert, H. E. \& Simpson, J. E. 1980 The slumping of gravity currents. J. Fluid Mech. 99, 785-799.

Kneller, B., Bennett, S. J. \& MCCAfFrey, W. D. 1999 Velocity structure, turbulence and fluid stresses in experimental gravity currents. J. Geophys. Res. 104, 5281-5291.

Marino, B. M., Thomas, L. P. \& Linden, P. F. 2005 The front condition for gravity currents. J. Fluid Mech. 536, 49-78.

RotTMAn, J. W. \& Simpson, J. E. 1983 Gravity currents produced by instantaneous releases of a heavy fluid in a rectangular channel. J. Fluid Mech. 135, 95-110.

SHER, D. \& WoOdS, A. W. 2015 Gravity currents: entrainment, stratification and self-similarity. J. Fluid Mech. 784, 130-162.

Shin, J. O., Dalziel, S. B. \& Linden, P. F. 2004 Gravity currents produced by lock exchange. J. Fluid Mech. 521, 1-34. 